\title{
RCT vs register
}

\author{
Marco Cenzato $^{1}$ (D) Edoardo Boccardi $^{2}$
}

Received: 17 July 2017 / Accepted: 19 July 2017 /Published online: 8 August 2017

(C) Springer-Verlag GmbH Austria 2017

\section{Dear Editor,}

Thank you for your thorough analysis and thoughtful conclusions. We are happy to see that we share the same concerns about the Aruba study, i.e. that it did not provide answers to the dilemma of treating arteriovenous malformation patients in terms of who, how and when, but rather it created further ethical and legal problems. That was exactly the basis on which the European Consensus Conference was initiated and we are also glad that you support most of its proposals.

As you pointed out, the TOBAS study consists of two components: one is a register and the other one is a randomised trial.
While we obviously agree that randomisation is the best way to achieve scientifically pure data, we see the many difficulties and limitations of such an approach, not least the loss of the data coming from all the cases excluded from the study (200 included and 1,700 excluded in the Aruba study).

We opted, therefore, to conduct a most comprehensive register, but being a European initiative we would rather gather the information locally. Naturally there is no reason why the data from the two registers should not subsequently be assembled and evaluated as a whole. The more data the better, but the data collections would need to be homogeneously comparable.

Marco Cenzato

marco.cenzato@gmail.com

1 Department of Neurosurgery, Grande Ospedale Maggiore Niguarda, Piazza Ospedale Maggiore 3, 20162 Milan, Italy

2 Department of Neuroradiology, Grande Ospedale Maggiore Niguarda, Piazza Ospedale Maggiore 3, 20162 Milan, Italy 